\title{
FOXF2 deficiency promotes epithelial-mesenchymal transition and metastasis of basal-like breast cancer
}

Qing-Shan Wang ${ }^{1,2+}$, Peng-Zhou Kong ${ }^{1 \dagger}$, Xiao-Qing Li ${ }^{1,2}$, Fan Yang ${ }^{1}$ and Yu-Mei Feng ${ }^{1,2^{*}}$

\begin{abstract}
Introduction: Our previous clinical study demonstrated that the under-expression of FOXF2 is associated with earlyonset metastasis and poor prognosis of patients with triple-negative breast cancer. In this study, we further characterized the role of FOXF2 in metastasis of basal-like breast cancer (BLBC) and underlying molecular mechanisms.

Methods: RT-qPCR, immunoblot, immunofluorescence and immunohistochemistry were performed to assess the expression of genes and proteins in cell lines and tissues. A series of in vitro and in vivo assays was performed in the cells with RNAi-mediated knockdown or overexpression to elucidate the function and transcriptional regulatory role of FOXF2 in breast cancer.
\end{abstract}

Results: We found that FOXF2 was specifically expressed in most basal-like breast cells. FOXF2 deficiency enhanced the metastatic ability of BLBC cells in vitro and in vivo. Additionally, FOXF2 deficiency induced the epithelial-mesenchymal transition (EMT) of basal-like breast cells. Furthermore, we identified that TWIST1 is a transcriptional target of FOXF2. TWIST1 was negatively regulated by FOXF2 and mediated the FOXF2-regulated EMT phenotype of basal-like breast cells and aggressive property of BLBC.

Conclusions: FOXF2 is a novel EMT-suppressing transcription factor in BLBC. FOXF2 deficiency enhances metastatic ability of BLBC cells by activating the EMT program through upregulating the transcription of TWIST1.

\section{Introduction}

Breast cancer is the most frequently diagnosed cancer among females [1]. Metastasis is the leading cause of death in breast cancer patients. To develop distant metastatic disease, the epithelial cancer cells need to undergo a morphological change into a mesenchymal phenotype to acquire migration and invasion ability [2]. Epithelialmesenchymal transition (EMT) is a program of switching polarized epithelial cells to migratory mesenchymal cells [2]. The aberrant activation of the EMT program is implicated in tumor progression, metastasis and acquisition of therapeutic resistance [2,3]. EMT-transcription

\footnotetext{
* Correspondence: ymfeng@tijmu.edu.cn

${ }^{\dagger}$ Equal contributors

'Department of Biochemistry and Molecular Biology, Tianjin Medical University Cancer Institute and Hospital, National Clinical Research Center of Cancer, Huan-Hu-Xi Road, Tianjin 300060, China

${ }^{2}$ Key Laboratory of Breast Cancer Prevention and Treatment of the Ministry of Education, Tianjin Medical University Cancer Institute and Hospital,

National Clinical Research Center of Cancer, Huan-Hu-Xi Road, Tianjin 300060, China
}

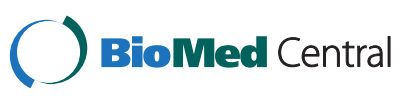

(c) 2015 Wang et al.; licensee BioMed Central. This is an Open Access article distributed under the terms of the Creative Commons Attribution License (http://creativecommons.org/licenses/by/4.0), which permits unrestricted use, distribution, and reproduction in any medium, provided the original work is properly credited. The Creative Commons Public Domain Dedication waiver (http://creativecommons.org/publicdomain/zero/1.0/) applies to the data made available in this article unless otherwise stated. factors (EMT-TFs) such as TWIST1, SNAIL1, SNAIL2, ZEB1, ZEB2, Goosecoid and FOXC2, can trigger the EMT program by directly or indirectly suppressing Ecadherin expression [4-7]. TWIST1 is a member of the basic helix-loop-helix (bHLH) transcription factor family [8] and plays a pivotal role in the regulation of embryogenesis, gastrulation and mesoderm formation during early embryonic development $[9,10]$. TWIST1 was found to be frequently activated in a wide array of human cancers and is associated with poor prognosis [3,11]. TWIST1 induces the EMT program by downregulating E-cadherin expression through indirect effects on the $C D H 1$ promoter [3].

Breast cancer is a heterogeneous disease. Based on their gene expression profiles, breast cancers can be classified into distinct molecular subtypes: normal breast-like, luminal A, luminal B, human epidermal growth factor receptor 2 (HER2)-enriched, and basal-like [12]. Basal-like breast cancer (BLBC) is less likely to express estrogen receptor (ER), progesterone receptor (PR) and HER2, which 
are also characteristics of triple-negative breast cancer (TNBC). Thus, BLBC shares many features with TNBC, and the two terms are often used interchangeably [13]. In addition to the triple-negative marker status, BLBC is characterized by the expression of basal markers such as cytokeratins (CK) 5/6, CK14, CK17 and epithelial growth factor receptor (EGFR) in the clinic [12]. TNBC and/or BLBC is recognized as a particularly aggressive subtype and receives less benefit from targeted therapy [12]. Therefore, there is an urgent need to elucidate the molecular pathogenesis of TNBC and/or BLBC and develop effective systemic therapies, especially molecular-targeted therapy.

Recent reports have revealed that TNBC/BLBC is a group of heterogeneous tumors [14]. BLBC also can be divided into extraordinarily diverse basal-like $\mathrm{A}$ and basal-like B subtypes [15]. The basal-like A cells have either luminal-like or basal-like epithelial morphology, while the basal-like B cells appear poorly differentiated and possess more mesenchymal characteristics [15]. Thus, the basal-like B subtype is more aggressive than the basal-like A subtype [15]. Due to the heterogeneity of BLBC, it is important to identify the critical regulatory factors that are associated with aggressive phenotype of BLBC. It is well known that various embryonic and mesenchymal EMT-TFs, including SNAIL1 [16], SNAIL2 [17] TWIST1 [18], and Forkhead box (FOX) transcription factor superfamily members FOXC1 [19], FOXC2 [4] and FOXQ1 [20], contribute to the aggressive phenotype of BLBC.

The mesenchymal regulator FOXF2 belongs to the FOX transcription factor superfamily [21]. It is specifically expressed in the mesenchyme adjacent to the epithelium in organs derived from the splanchnic mesoderm [22], and plays an important role in tissue homeostasis through regulating epithelium-mesenchyme interaction to maintain epithelium polarity [22]. Our previous clinical study demonstrated that the under-expression of FOXF2 is associated with early-onset metastasis and poor prognosis of patients with TNBC, but not the prognosis of non-TNBC patients [23]. This result suggests that FOXF2 deficiency is involved in TNBC/BLBC metastasis through regulating EMT. Recent studies have indicated that FOXF2 is a potential tumor suppressor in both prostate cancer [24] and breast cancer [25]. However, the role of FOXF2 in breast cancer metastasis and the underlying molecular mechanisms remain largely unknown.

In this study, we identified FOXF2 as a novel EMTsuppressing transcription factor in BLBC and demonstrated that it directly represses the transcription of TWIST1. FOXF2 deficiency promotes metastasis of BLBC cells through transcriptional upregulation of TWIST1 and activation of EMT.

\section{Materials and methods \\ Cell culture}

The human breast cancer cell lines MDA-MB-231, BT549, MCF-7, BT474, ZR-75-30, SKBR-3 and MDAMB-453, immortalized non-tumorigenic basal-like mammary epithelial cell lines MCF-10A and HBL100 were obtained from American Type Culture Collection (Manassas, VA, USA). MDA-MB-231-luc-D3H2LN (231Luc), a MDA-MB-231 subclone expressing luciferase, was obtained from Caliper Life Sciences (Hopkinton, MA, USA). MCF-10A cells were maintained in DMEM-F12 medium (Invitrogen, Gaithersburg, MD, USA) supplemented with $5 \%$ horse serum, $20 \mathrm{ng} / \mathrm{mL}$ EGF, $500 \mathrm{ng} / \mathrm{mL}$ hydrocortisone, $10 \mu \mathrm{g} / \mathrm{mL}$ insulin and $100 \mathrm{ng} / \mathrm{mL}$ cholera toxin. The other cells were cultured in DMEM-F12 (MCF-7 and MDA-MB-453) or RPMI 1640 (MDA-MB231, BT549, BT474, ZR-75-30, SKBR-3 and HBL100) medium (Invitrogen) supplemented with 10\% fetal bovine serum (FBS; Invitrogen), 100 units $/ \mathrm{mL}$ penicillin, and $100 \mathrm{mg} / \mathrm{mL}$ streptomycin (Invitrogen). All cell lines were incubated in a humidified incubator at $37^{\circ} \mathrm{C}$ with $5 \% \mathrm{CO}_{2}$ and grown into logarithmic phase and/or $80 \%$ confluence for the experiments.

\section{Lentiviral transduction of shRNA and transfection of interfering RNA and plasmids}

To obtain the stable FOXF2-knockdown cells, cells were infected with the lentiviral vector expressing a short hairpin RNA (shRNA) targeting the sequence GTCCTC AACTTCAATGGGATT in the coding sequence region of human FOXF2 gene (shFOXF2; Sigma-Aldrich, St Louis, MO, USA; TRCN0000013959). A shRNA nontargeting human and mouse gene was used as control (shControl; Sigma-Aldrich; SHC002). The cells were selected in $2 \mu \mathrm{g} / \mathrm{mL}$ puromycin to establish stable expressing shRNAs cells. For the rescue experiment, the stable FOXF2-knockdown cells were transfected with shRNAresistant full-length FOXF2 plasmid (Genechem, Nanjin, China), which has three nucleotide mismatches within the target sequence of the shFOXF2 (GTCCTCAATTT TAACGGGATT). To silence the expression of TWIST1, the cells were transiently transfected with two independent small interfering RNAs (siRNAs) targeting the coding sequence region of human TWIST1 gene GGATCA AACTGGCCTGCAA (si-TW\#1) and GAACACCTTTA GAAATAAA (si-TW\#2; RiboBio Co., Guangzhou, China) or a negative control siRNA (si-Control) using Lipofectamine 2000 (Invitrogen) according to the manufacturer's specifications. The human full-length FOXF2 cDNA was amplified from a pEV-FOXF2 plasmid, which was a gift from Professor Peter Carlsson (University of Gothenburg, Department of Cell and Molecular Biology, Sweden), and then was subcloned into the pcDNA3.1-HA vector (Invitrogen). The human full-length TWIST1 cDNA (Genechem) 
was subcloned into the pcDNA3.1 vector (Invitrogen). The cells were transfected with pcDNA3.1-HA-FOXF2 (FOXF2), pcDNA3.1-TWIST1 (TWIST1) or vector control (Invitrogen) using Lipofectamine 2000.

\section{Reverse transcription quantitative polymerase chain reaction}

Total RNA isolation from cultured cells or tissues, reverse transcription (RT) reactions, quantitative PCR (qPCR), and the quantification of target gene expression were performed as previously described [23].

\section{Immunoblot, immunohistochemistry, and immunofluorescence}

The protocols for protein analysis by immunoblot, immunohistochemistry, and immunofluorescence were performed as previously described [26]. The primary antibodies were anti-E-cadherin, anti-N-cadherin, antivimentin (BD Biosciences, Bedford, MA, USA), antiFOXF2 (Abnova, Aachen, Germany), anti-TWIST1, anti-HA tag (Abcam, Cambridge, MA, USA), and anti- $\beta$ actin (Sigma-Aldrich).

\section{Chromatin immunoprecipitation assay}

Chromatin immunoprecipitation (ChIP) assay was performed using a ChIP assay kit (EMD Millipore, Billerica, MA, USA) according to the manufacturer's instructions. Anti-HA antibody (Abcam) was used for immunoprecipitation to enrich the promoter fragments containing putative FOXF2 binding site of target genes in MCF-10A cells constitutively expressing HA-tagged FOXF2 (MCF10A-FOXF2). The isotype IgG (Abcam) was used as a negative control. The primers for the amplification of the TWIST1 promoter region containing a putative FOXF2 binding site from -2120 to -2113 relative to the transcription start site (TSS) were 5'-AGATTTCCTT TACACTTTACCC-3' (forward) and 5'-GCGAGTGTT ATTTCTCCAGCGA-3' (reverse). The primers for the amplification of TWIST1 exon $1(+408$ to +841), which served as a negative control, were $5^{\prime}$-CAGCGAGGAA GAGCCAGACCG-3' (forward) and 5'-GGAGGACCTG GTAGAGGAAGT-3' (reverse).

\section{Luciferase reporter assay}

The TWIST1 promoter region with $(-2140$ to +27$)$ or without $(-2057$ to +27$)$ FOXF2 binding sites was amplified from human genomic DNA using the primers $5^{\prime}$ AGTCCAATCATTCGATCTC-3' (forward) and 5' -CTG CAGACTTGGAGGCT-3' (reverse), or 5'-ATAGCTGA AGTGGAAAAGG-3' (forward) and 5' -CTGCAGACTT GGAGGCT-3' (reverse) with Kpn I and Bgl II restriction endonuclease recognition sites at the $5^{\prime}$-ends, respectively. The fragments of the TWIST1 promoter were cloned into the luciferase reporter gene plasmid pGL3-
Basic (Promega, Madison, WI, USA). The pGL3 reporter and pRL-TK plasmid were transiently co-transfected into MCF-10A and BT549 cells for $48 \mathrm{~h}$. The luciferase activity of pGL3-TWIST1 (-2140/+27) or pGL3-TWIST1 (-2057/ +27 ) was normalized to Renilla luciferase activity.

\section{Cell proliferation}

Cell proliferation ability was assessed using a 3-(4,5dimethylthiazol-2-yl)-2,5-diphenyltetrazolium bromide (MTT) assay. Cells were seeded at a density of $2 \times 10^{3}$ cells/well in 96-well plates. On day $1,2,3,4$ and 5 , the cells were incubated with $10 \mu \mathrm{L}$ MTT solution $(5 \mathrm{mg} / \mathrm{mL}$ in phosphate-buffered saline (PBS)) at $37^{\circ} \mathrm{C}$ for $4 \mathrm{~h}$. After removal of the medium, $100 \mu \mathrm{L}$ DMSO was added to each well and absorbance was measured at $570 \mathrm{~nm}$.

\section{Cell invasion and migration assay}

Cell invasion and migration ability in vitro were assessed using Matrigel-coated and non-Matrigel-coated transwell inserts (BD Biosciences) as described previously [26].

\section{Xenograft tumor assay}

Twenty female 4- to 6-week-old severe combined immunodeficiency (SCID) mice were randomly divided into two groups for the xenograft tumor assay. Then, $5 \times 10^{6}$ 231-Luc cells infected with shFOXF2 (231-Luc-shFOXF2) or shControl (231-Luc-shControl) were washed and harvested in $0.1 \mathrm{~mL}$ PBS and injected into the left lower abdominal mammary fat pad of the SCID mice [27]. After 22 days, all mice were anesthetized using isoflurance and injected intraperitoneally with $150 \mathrm{mg} / \mathrm{kg}$ D-luciferin/PBS. The bioluminescent imaging was performed using a Xenogen IVIS system (Caliper Life Sciences, Hopkinton, MA, USA). The data of living image were expressed as photon flux (photons per second). The tumor volumes were measured by caliper at the indicated time and calculated using the formula (length $\mathrm{x}$ width ${ }^{2}$ )/2 [28]. The survival time of mice was defined as the time interval between the day of cell injection and the time of death or sacrifice. The xenograft tumors, livers and lungs were harvested from xenograft mice at sacrifice. The tissues were prepared for paraffin-embedded tissue section and hematoxylin and eosin (H\&E) staining or immunohistochemistry staining. Two independent experiments were performed with xenograft mice. In one experiment, the mice died naturally to allow survival time calculations. In the second experiment, the mice were sacrificed for dissection on day 21 after cell injection. The mouse xenograft tumor assays were performed in accordance with protocols approved by the Animal Ethics Committee of Tianjin Medical University Cancer Institute and Hospital (TMUCIH; Tianjin, China. No. 058). 


\section{Breast cancer tissue specimens}

A total of 34 primary TNBC tissue specimens were obtained from patients who underwent breast surgery in TMUCIH. The use of these specimens was approved by the Institutional Review Board of TMUCIH, and written consent was obtained from all participants. All cases were followed up over 3 years, and 30 cases were followed up over 5 years. Disease-free survival (DFS) was defined as the time interval between primary surgery and any relapse (local-regional, contra-lateral and/ or distant), or the terminal time of follow-up without any relapse events.

Independent data sets for validation of gene expression levels in breast cancer cell lines and breast cancer tissues The FOXF2 mRNA expression pattern in different subtypes of breast cell lines was validated using the Gene expression-based Outcome for Breast cancer Online (GOBO) data sets [29].

\section{Statistical analysis}

Data from in vitro and in vivo experiments were presented as mean \pm standard deviation (SD). Student's $t$ test was used to compare differences between the experimental group and the control group. Fisher's exact test was used to compare the difference of metastatic incidence between 231-Luc-shFOXF2 mice and the 231Luc-shControl mice. Spearman's rank correlation was used to analyze the correlation between FOXF2 and TWIST1 mRNA levels in breast cancer tissues. Survival plots were created using Kaplan-Meier analysis, and logrank test was used to assess statistical significance. $P<0.05$ was considered statistically significant.

\section{Results}

FOXF2 deficiency enhances migration and invasion of basal-like mammary epithelial cells and BLBC cells in vitro FOXF2 mRNA and protein expression levels in various breast cell lines, including basal-like subtype cells MDAMB-231, BT-549, MCF-10A, HBL100 and non-basal-like cells MCF-7, BT474, ZR-75-30, SKBR-3 and MDA-MB453 , were measured by RT-qPCR and immunoblot, respectively. The results showed that FOXF2 was highly expressed in most basal-like cell lines and was less expressed in non-basal-like cell lines (Figure 1A and B). This expression pattern of FOXF2 in the basal-like ( $\mathrm{n}=14$ ) and non-basal-like (luminal; $\mathrm{n}=25$ ) subtype breast cell lines was validated using GOBO data set analysis (Figure 1C). The result confirmed that FOXF2 was frequently and highly expressed in mesenchymal/myoepithelium-like breast cells. Combining these results with our previous finding that the under-expression of FOXF2 is associated with early-onset metastasis and poor prognosis of TNBC patients, but not the prognosis of non-TNBC patients [23], we hypothesized that FOXF2 may play a role in the metastasis of BLBC.

To investigate the roles of FOXF2 in regulating the invasive potential of BLBC cells in vitro, the immortalized non-tumorigenic basal-like mammary epithelial cell line MCF-10A and the invasive BLBC cell lines MDA-MB231 was selected to establish stable FOXF2-knockdown cells by infection with a lentiviral vector expressing a FOXF2 shRNA (shFOXF2) or a non-target shRNA control lentiviral vector (shControl). The knockdown efficiency of FOXF2 in these cells was shown in Figures 1D and $2 \mathrm{~B}$. Boyden chamber transwell assays showed that FOXF2 knockdown significantly promotes migration and invasion of both the two cell lines (Figure 1E). To exclude the off-target effects of the shFOXF2, we performed a rescue experiment by transfecting a shRNA-resistant fulllength FOXF2 in the stable FOXF2-knockdown cells. The results showed that the effect of shFOXF2 on migration and invasion of MCF-10A and MDA-MB-231 cells was reversed (Figure 1E). Next, we transiently transfected pcDNA3.1-FOXF2 in the BT549 cells, a BLBC cell line with high invasiveness and less FOXF2 expression. The overexpressed FOXF2 in the cells was shown in Figures 1D and $2 \mathrm{~B}$. We observed that FOXF2 overexpression significantly decreased the migration and invasion of BT549 cells (Figure 1E). In contrast with the role of promoting cell migration and invasion, the cell growth curves showed that FOXF2 knockdown significantly suppressed the proliferation of MCF-10A and MDA-MB-231 cells and the transfection of shRNA-resistant full-length FOXF2 rescued the effect of shFOXF2 on the proliferation of MCF10A and MDA-MB-231 cells (Figure 1F). Conversely, FOXF2 overexpression in BT549 cells promoted the cell proliferation (Figure 1F). In addition, we also tested the functions of FOXF2 on luminal breast cancer cells by ectopically expressing FOXF2 in MCF-7 cells (see Figure S1A in Additional file 1). However, the exogenous FOXF2 did not significantly affect the migration, invasion and proliferation of MCF-7 cells compared to the control cells (see Figure S1B and C in Additional file 1). Collectively, these results suggest that FOXF2 deficiency enhances the metastatic potential of basal-like mammary epithelial cells and breast cancer cells but reduces cell proliferation.

\section{FOXF2 deficiency enhances metastatic ability of BLBC cells in vivo}

To confirm the role of FOXF2 in metastasis of BLBC cells in vivo, 231-Luc-shFOXF2 and 231-Luc-shControl cells were injected into the mammary fat pad of female SCID mice. The general physical condition of mice with 231-Luc-shFOXF2 cells (231-Luc-shFOXF2 mice) was weaker compared to that of mice with 231-Luc-shControl cells (231-Luc-shControl mice) by day 22 after injection (Figure 3A). The weight of 231-Luc-shFOXF2 mice was 
A

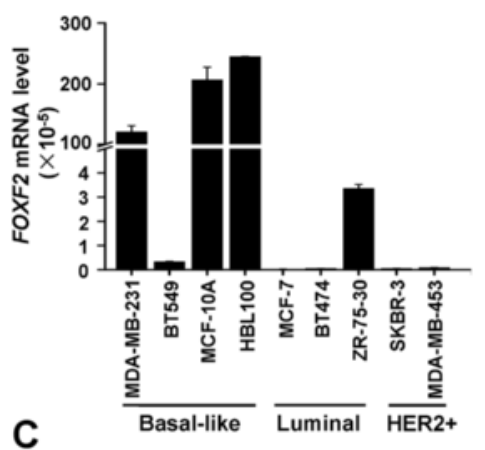

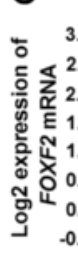

B

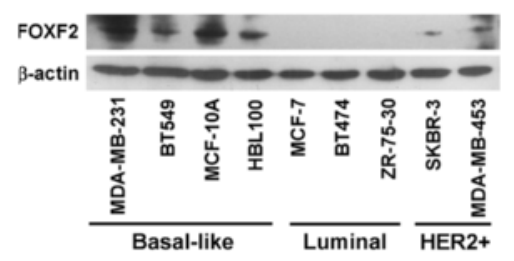

GOBO data
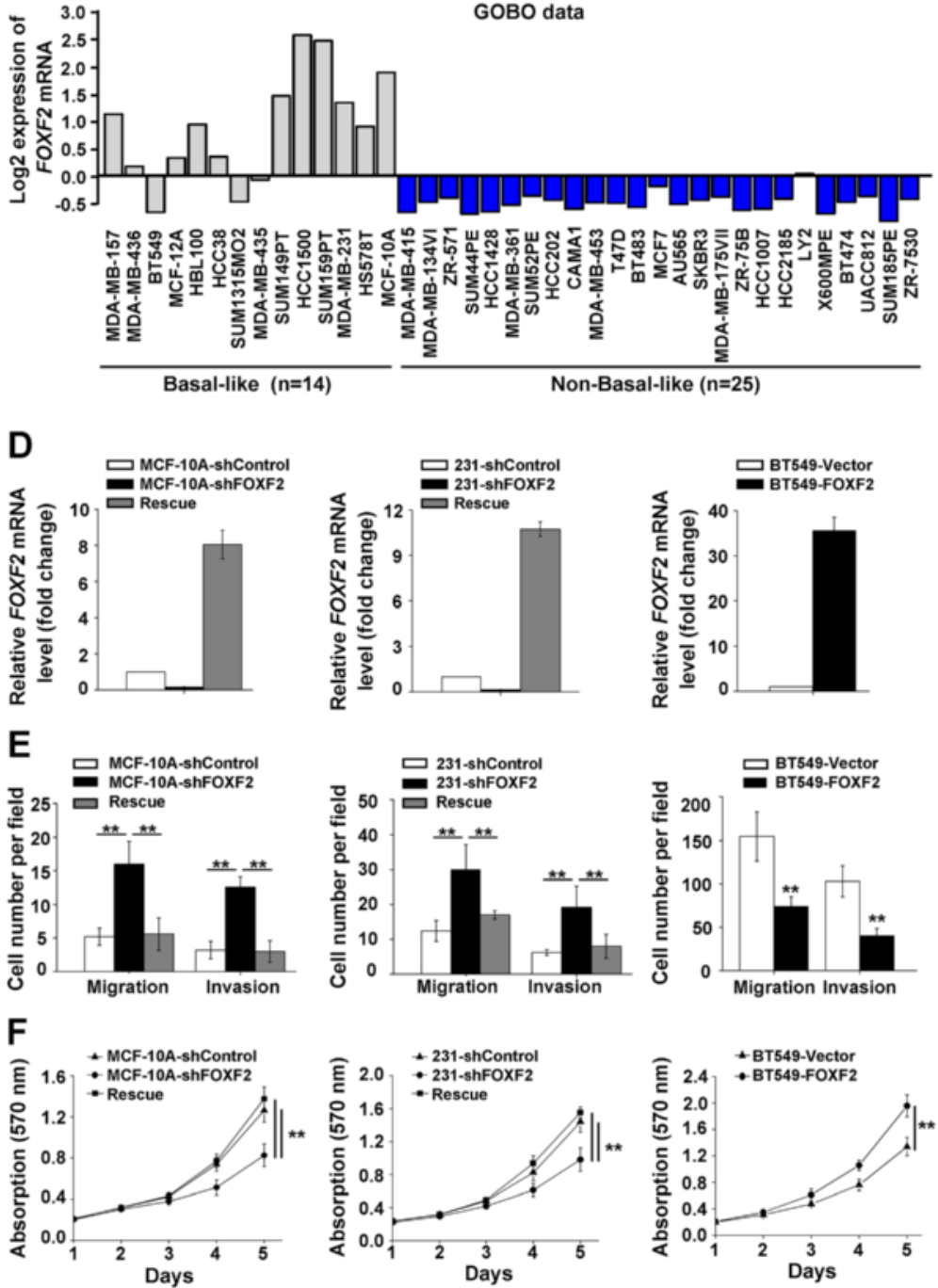

Figure 1 FOXF2 deficiency enhances migration and invasion of basal-like mammary epithelial cells and breast cancer cells in vitro. (A) FOXF2 mRNA levels in the indicated cell lines were measured by RT-qPCR. The data were normalized to the expression of the housekeeping gene glyceraldehyde 3-phosphate dehydrogenase (GAPDH). (B) The protein expression of FOXF2 in the indicated cells was detected by immunoblot. (C) FOXF2 mRNA levels in different subtypes of breast cell lines were analyzed using an independent GOBO dataset. (D) FOXF2 mRNA levels in the indicated cells were measured by RT-qPCR. Fold changes were relative to the mRNA expression of the control cells. (E) The migration and invasion ability of the indicated cells were assessed by transwell assay. (F) Proliferation of the indicated cells was determined by MTT assay. Three independent assays were performed in triplicate. The data were expressed as mean \pm SD. ${ }^{* *} P<0.01$. GOBO, Gene expression-based Outcome for Breast cancer Online; MTT, 3-(4,5-dimethylthiazol-2-yl)-2,5-diphenyltetrazolium bromide; RT-qPCR, reverse transcription quantitative PCR; SD, standard deviation. 


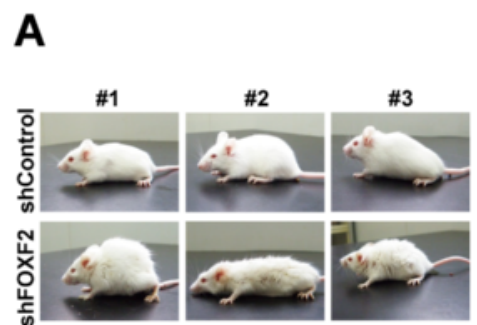

Day 22 after injection

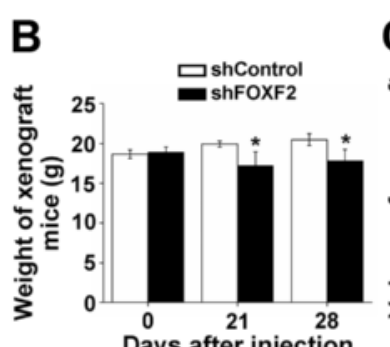

Days after injection

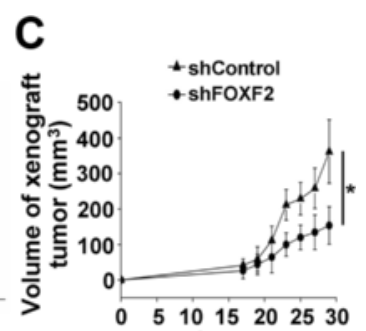

Days after injection

D

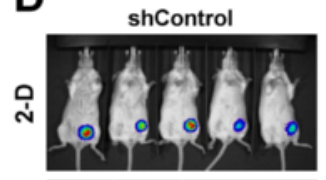

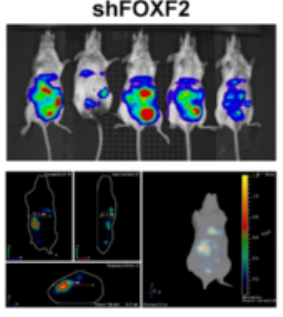

ay 22 after injection
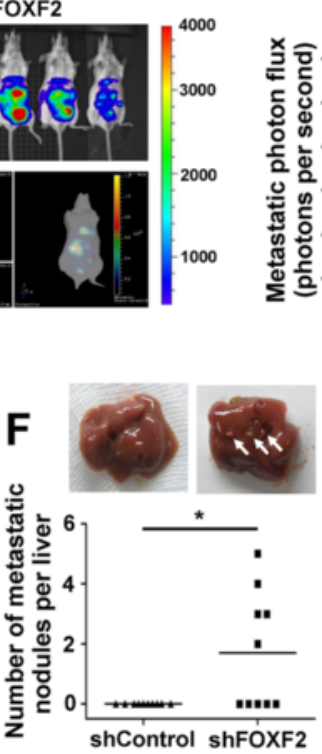

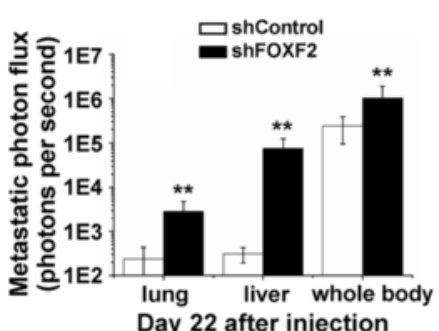

G

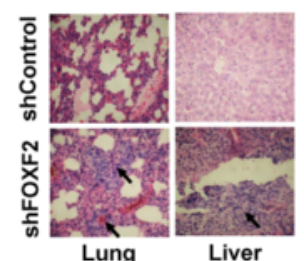

I

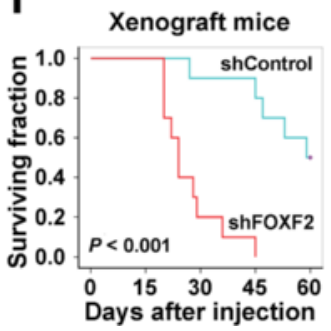

Figure 2 FOXF2 deficiency enhances metastatic ability of BLBC cells in vivo. (A) Representative photos of the general physical condition of xenograft mice injected with MDA-MB-231-Luc-shFOXF2 or the control cells on day 22 after injection. (B) The weights of xenograft mice injected with MDA-MB-231-Luc-shFOXF2 ( $n=4-10)$ or the control cells $(n=9-10)$ were measured at the indicated times. (C) Tumor volume of xenograft mice injected with MDA-MB-231-Luc-shFOXF2 $(n=3-10)$ or the control cells $(n=9-10)$ at the indicated times. (D) Bioluminescence images of primary xenograft tumor and metastatic tumor of xenograft mice injected with MDA-MB-231-Luc-shFOXF2 $(n=7)$ or the control cells $(n=10)$ on day 22 after injection (Left) and the quantitation of metastatic photon flux of the lung, liver and whole body (Right). (E) Metastatic incidence of xenograft mice injected with MDA-MB-231-Luc-shFOXF2 cells $(n=10)$ and the control cells $(n=10)$ on day 22 after injection. (F) Representative photos (Top) and count (Bottom) of visible metastatic nodules in the liver of euthanized xenograft mice injected with MDA-MB-231-Luc-shFOXF2 cells $(n=10)$ and the control cells $(n=10)$ on day 21 after injection. (G) Representative H\&E stained sections of lung and liver harvested from xenograft mice. The arrows point to the metastatic foci in lung and liver. (H) Representative H\&E-stained sections of primary tumor harvested from xenograft mice. The arrows indicate that the cancer cells invaded into muscle (Left) and vasculature (Right). The selected areas of muscular and vascular invasion are enlarged in the respective bottom panels. (I) Kaplan-Meier curves of the overall survival of xenograft mice ( $n=10)$. Two independent experiments were performed. The data were expressed as mean $\pm \mathrm{SD} .{ }^{*} P<0.05 ;{ }^{* *} P<0.01$. BLBC, basal-like breast cancer; H\&E, 
A
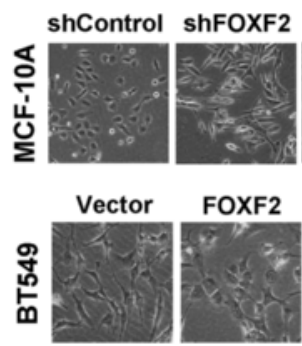

B

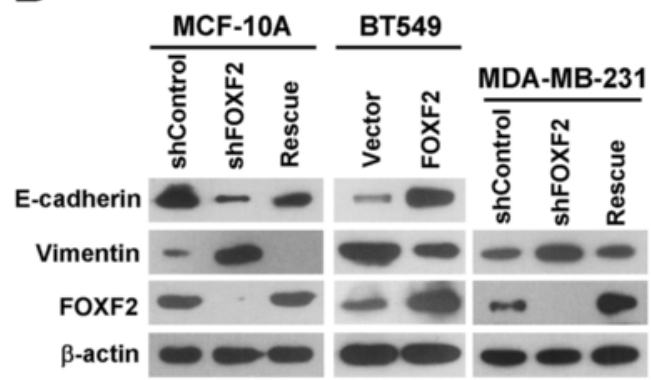

C

E-cadherin $\beta$-catenin Vimentin Fibronectin
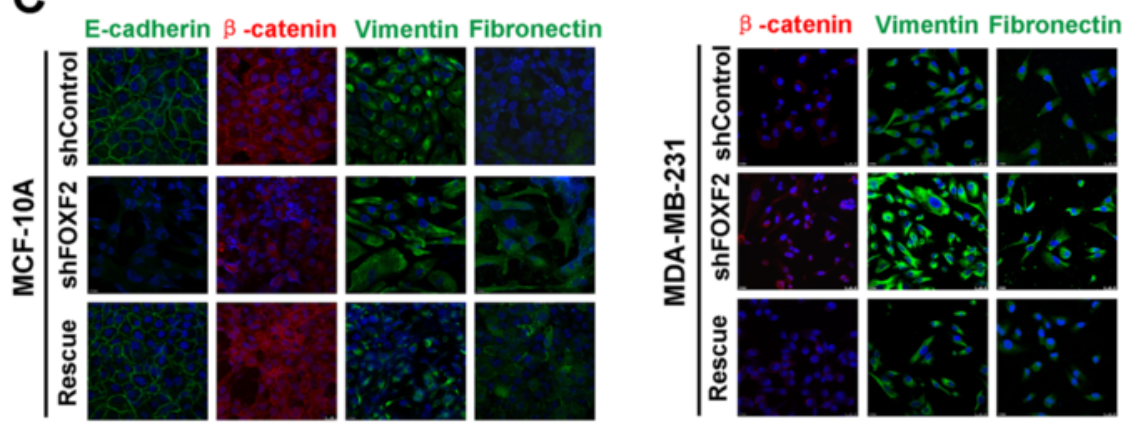

D

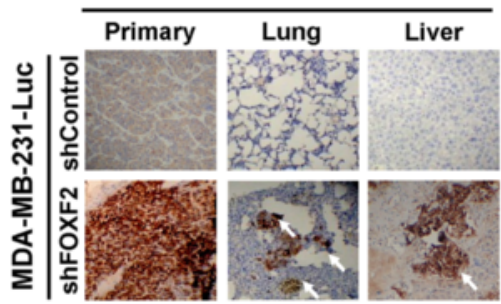

E

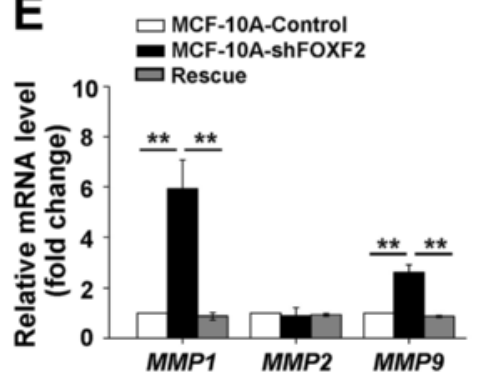

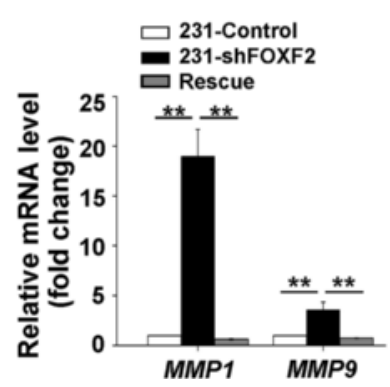

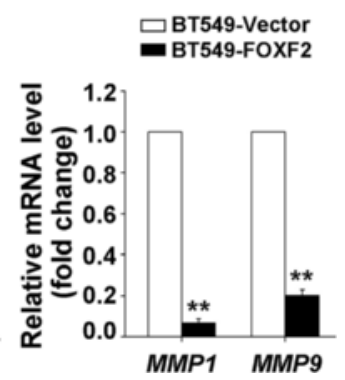

Figure 3 FOXF2 deficiency induces EMT phenotype of basal-like mammary epithelial cells and BLBC cells. (A) Morphological photos of the indicated cells (X200). (B) The protein expression of EMT markers and FOXF2 in the indicated cells was detected by immunoblot. (C) The protein expression of EMT markers in the indicated cells was detected by immunofluorescence staining. (D) Vimentin expression in tissues of primary tumor, lung metastases and liver metastases harvested from xenograft mice were stained by immunohistochemistry $(\times 200)$. Arrows point to the metastatic cancer cells. (E) MMP1, MMP2 and MMP9 mRNA levels in the indicated cells were measured by RT-qPCR. Fold changes were relative to the mRNA expression of the control cells. Three independent assays were performed in triplicate. The data were expressed as mean \pm SD. ${ }^{* *} P<0.01$. BLBC, basallike breast cancer; EMT, epithelial-mesenchymal transition; RT-qPCR, reverse transcription quantitative PCR; SD, standard deviation.

also lower than that of the 231-Luc-shControl mice (Figure 3B). Consistent with the cell proliferation experiments in vitro, 231-Luc-shFOXF2 mice had smaller xenograft tumors than the 231-Luc-shControl mice (Figure 3C).
At day 22 post injection, we performed in vivo bioluminescent imaging to monitor the metastasis using a Xenogen IVIS system. The bioluminescent imaging and metastatic photon flux analysis revealed that the 231- 
Luc-shFOXF2 mice suffered significantly more local invasion and metastatic dissemination than the 231-LucshControl mice, especially in the lung and liver metastases (Figure 3D). The data revealed that $80 \%$ of 231 -LucshFOXF2 mice (8/10) suffered metastasis, whereas, $20 \%$ of the control mice $(2 / 10)$ suffered metastasis (Figure 3E). Visible liver metastatic nodules were found in half of the 231-Luc-shFOXF2 mice (5/10), whereas none were found in the livers of the 231-Luc-shControl mice (Figure 3F). H\&E staining confirmed that more metastatic foci formed in the lungs and livers in 231-Luc-shFOXF2 mice than in the control mice (Figure 3G). Moreover, there was more muscle invasion in 231-Luc-shFOXF2 mice than in the control mice. The tumors had blood vessel invasion in the xenograft tumors of 231-Luc-shFOXF2 mice but not in the tumors of the control mice (Figure 3H). A Kaplan-Meier survival analysis revealed that the 231Luc-shFOXF2 mice had a significantly shorter survival time than the 231-Luc-shControl mice (Figure 3I). Taken together, these results indicate that FOXF2 deficiency enhances the metastatic ability of BLBC cells and leads to invasive and metastatic disease in vivo.

\section{FOXF2 deficiency induces EMT phenotype of basal-like mammary epithelial cells and BLBC cells}

During mouse embryogenesis, FOXF2 is expressed in the mesenchyme derived from the splanchnic mesoderm adjacent to the ectoderm-derived epithelium and plays an important role in tissue homeostasis through regulating epithelium-mesenchyme interactions to maintain the epithelium polarity [22]. This expression pattern suggests a potential role of FOXF2 in regulating EMT. Therefore, we hypothesized that FOXF2 deficiency in BLBC metastasis might be involved the EMT process. We next investigate whether FOXF2 deficiency induces EMT in BLBC cells. Compared with the MCF-10AshControl cells, FOXF2 knockdown transformed MCF10A cells from epithelial morphology into fibroblast-like shape and caused cell scattering, and the expression of shRNA-resistant full-length FOXF2 rescued the phenotype (Figure 2A). The detection of EMT markers revealed that, compared to the control cells, FOXF2knockdown cells had reduced level of the epithelial marker E-cadherin in MCF-10A cells (undetectable in the MDA-MB-231 cell lines). Additionally, the FOXF2knockdown cells had elevated levels of the mesenchymal markers vimentin in the MCF-10A, MDA-MB-231 cell lines (Figure 2B). Immunofluorescence staining revealed that the epithelial markers E-cadherin (undetectable in MDA-MB-231) and $\beta$-catenin were lost from cell membranes of FOXF2-knockdown cells and that the mesenchymal markers vimentin and fibronectin were increased (Figure 2C). The expression of shRNA-resistant fulllength FOXF2 reversed the effect of shFOXF2 on the expression of EMT markers (Figure $2 \mathrm{~B}$ and $\mathrm{C}$ ). Immunohistochemistry staining confirmed that vimentin was increased in primary xenograft tumors of 231-LucshFOXF2 mice and metastases in lungs and livers (Figure 2D). FOXF2 knockdown upregulated the expression of MMP1 and MMP9 in MCF-10A, MDA-MB-231, and the expression of shRNA-resistant full-length FOXF2 rescued the effect of shFOXF2 (Figure 2E). Conversely, FOXF2 overexpression in BT549 cells resulted in a reversal of the EMT phenotype (Figure $2 \mathrm{~A}, \mathrm{~B}$ and $\mathrm{E}$ ). However, the ectopic expression of FOXF2 in MCF-7 cells did not significantly affect the morphology (see Figure S1D in Additional file 1) and the expression of E-cadherin and vimentin (see Figure S1E in Additional file 1). These observations indicate that FOXF2 functions as an EMT suppressor in basal-like breast cells. FOXF2 deficiency enhances metastatic ability of basallike mammary epithelial cells and BLBC cells through inducing an EMT phenotype.

\section{FOXF2 deficiency activates EMT program of basal-like mammary epithelial cells and BLBC cells through upregulation of EMT-TFs}

To further investigate the mechanisms of how FOXF2 deficiency induces EMT phenotype, we examined the mRNA expression of EMT-TFs including SNAIL1, SNAIL2, TWIST1, ZEB1, ZEB2 and GSC (coding gene of Goosecoid) in MCF-10A, MDA-MB-231 cells with or without FOXF2 knockdown. The results showed that FOXF2 knockdown upregulated TWIST1 and ZEB2 expression in both MCF-10A and MDA-MB-231 cells (Figure 4A and B). Only ZEB1 and GSC were upregulated in MDA-MB-231 cells and SNAIL2 was induced in MCF-10A cells (Figure 4A and B). And the expression of shRNA-resistant full-length FOXF2 rescued the effect of shFOXF2 (Figure 4A and B). Conversely, FOXF2 overexpression in BT549 cells downregulated SNAIL2, TWIST1, $Z E B 1$, and GSC expression (Figure 4C). However, the ectopic expression of FOXF2 in MCF-7 cells did not significantly change the expression of these EMT-TFs (see Figure S1F in Additional file 1). These results indicate that FOXF2 deficiency induced the EMT phenotype through the activation of different EMT-TFs in the individual basal-like breast cell lines.

\section{FOXF2 binds to TWIST1 promoter and suppresses its transcription}

Because TWIST1 mRNA expression levels were commonly and negatively correlated with FOXF2 expression in the basal-like breast cell lines (Figure 4A, B and C), we investigated whether TWIST1 is a transcriptional target of FOXF2. First, we performed a BLAST search for FOXF2 binding sites in the promoter region of the TWIST1 gene. We found a putative FOXF2 binding site 

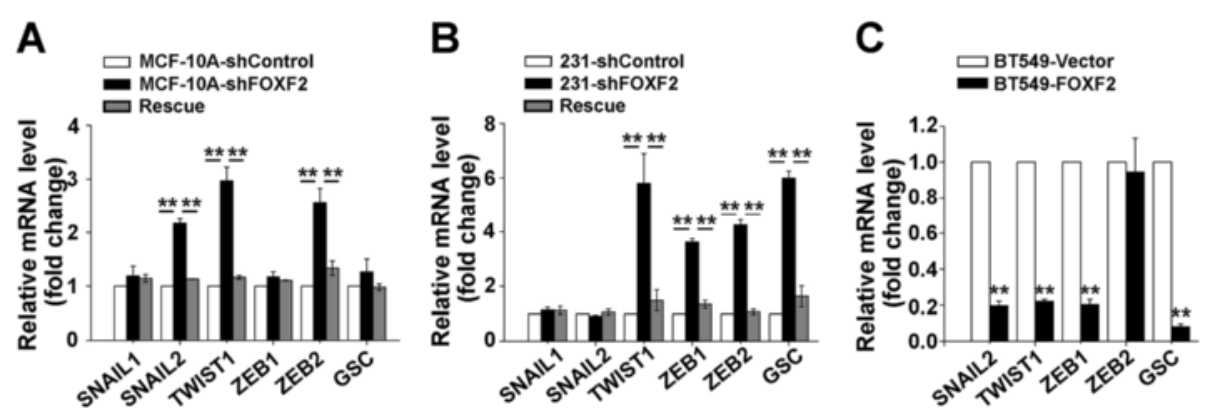

Figure 4 FOXF2 deficiency activates EMT programming through upregulating EMT-TFs. (A, B and C) The mRNA expression levels of EMTTFS SNAIL1, SNALI2, TWIST1, ZEB1, ZEB2 and GSC in the indicated cells were measured by RT-qPCR. Fold changes were relative to the mRNA expression of the control cells. Three independent assays were performed in triplicate. The data were expressed as means \pm SD. ${ }^{*} P<0.05 ;{ }^{* * *} P<0.01$. EMT, epithelial-mesenchymal transition; EMT-TFs, EMT-transcription factors; RT-qPCR, reverse transcription quantitative PCR; SD, standard deviation.

AATAAATA in the TWIST1 promoter region from -2120 to -2113 relative to the transcription start site (TSS; Figure 5A). Subsequently, the binding of FOXF2 on the TWIST1 promoter region containing or lacking the putative binding site was verified by ChIP assay in MCF-10A cells. The results confirmed that FOXF2 can bind to the TWIST1 promoter region containing the putative binding site but cannot bind to region lacking the putative binding site (Figure 5B). Therefore, TWIST1 is a candidate transcriptional target gene of FOXF2.

To further assess the regulatory activity of FOXF2 on the TWIST1 promoter, we performed a luciferase reporter assay by transfecting pGL3-TWIST1 (-2140 to +27) containing the putative binding site or pGL3TWIST1 (-2057 to +27) lacking the putative binding site in MCF-10A cells with or without FOXF2 knockdown. The results showed that the reporter activity of pGL3-TWIST1 (-2140 to +27) was increased in MCF10A-shFOXF2 cells compared to the control cells, and the expression of shRNA-resistant full-length FOXF2 rescued the effect of shFOXF2 (Figure $5 \mathrm{C}$ ). The reporter activity of pGL3-TWIST1 (-2057 to +27) was not significantly changed in FOXF2-knockdown cells compared to the control cells (Figure 5C). Conversely, FOXF2 overexpression in BT549 cells decreased the reporter activity of pGL3-TWIST1 ( -2140 to +27 ; Figure 5C). Furthermore, the role of FOXF2 in negatively regulating TWIST1 protein expression was confirmed using FOXF2 loss or gain of function in MCF-10A and BT549 cells by immunoblot (Figure 5D) and immunofluorescence detection (Figure 5E). Whereas, the ectopic expression of FOXF2 in MCF-7 cells did not significantly change the reporter activity of pGL3-TWIST1 (see Figure S1G in Additional file 1) and TWIST1 expression (see Figure S1E in Additional file 1). These results demonstrate that FOXF2 suppress the transcriptional activity of the TWIST1 promoter in basal-like breast cells. Cumulatively, these data demonstrate that TWIST1 is a downstream target of FOXF2 and that FOXF2 suppresses TWIST1 promoter activity and protein expression in basal-like breast cells.

FOXF2 deficiency induces EMT phenotype of basal-like mammary epithelial cells and aggressive property of BLBC cells via upregulating TWIST1 expression

To further investigate whether TWIST1 mediates the role of FOXF2 deficiency in inducing EMT in basal-like breast cells, we knocked down TWIST1 expression in MCF-10A-shFOXF2 cells by transiently transfecting two independent TWIST1 siRNAs (si-TW\#1 and si-TW\#2), respectively. The results showed that the FOXF2 knockdown-induced EMT phenotype of MCF-10A cells was reversed by silencing TWIST1 expression. Also, the expression of E-cadherin and vimentin (Figure 6A) was reversed, and epithelial morphology was restored (Figure 6B). Additionally, the cells showed decreased migration and invasion (Figure 6C). Next, we transiently transfected pcDNA3.1-TWIST1 into MDA-MB-231 cells, and the overexpressed TWIST1 in the cells was shown in Figure 6D. We observed that TWIST1 overexpression resulted in elevated levels of the mesenchymal markers vimentin (Figure 6D) and enhanced migration and invasion of the cells (Figure 6E). These results suggested that TWIST may mediate FOXF2-regulated EMT phenotype and aggressive property.

To provide in vivo evidence to illustrate the effect of FOXF2 on metastasis was mediated by negatively regulating TWIST1 expression, we detected the TWIST1 protein expression in primary tumors formed by inoculation of MDA-MB-231-Luc shFOXF2 and MDA-MB-231-Luc shControl cells in SCID mice by immunohistochemistry staining. The result showed increased TWIST1 expression in xenograft tumor of MDA-MB-231-Luc shFOXF2 comparing to the control tumor (Figure 6F). Furthermore, we detected the mRNA levels of FOXF2 and TWIST1 mRNA in 34 cases of human primary TNBC tissues by RTqPCR. Consistent with the negative regulation of FOXF2 on the transcription of TWIST1, TWIST1 mRNA levels 

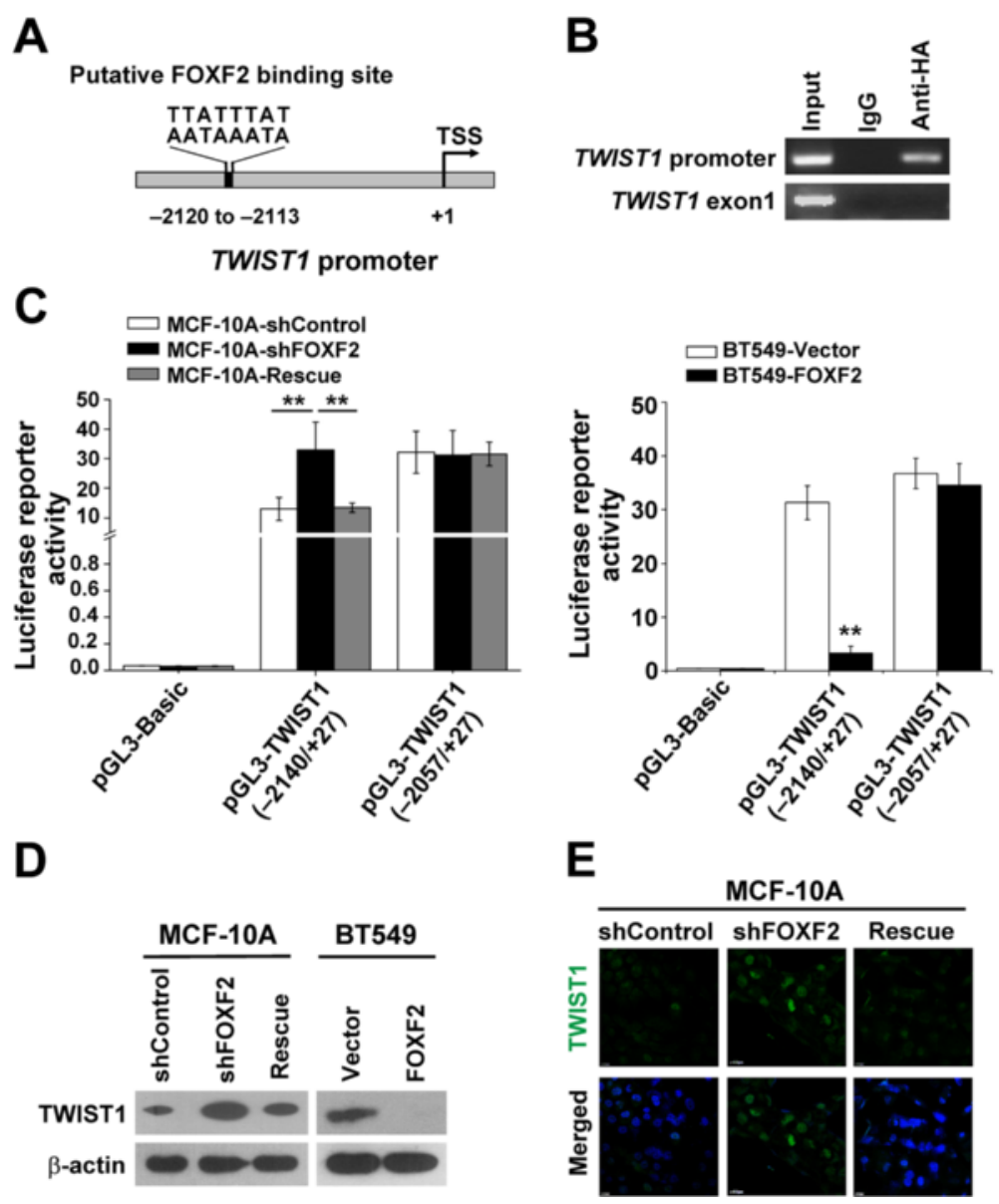

Figure 5 FOXF2 binds to TWIST1 promoter and suppresses TWIST1 transcription. (A) The putative binding site of FOXF2 on the TWIST 1 promoter region. (B) The binding of FOXF2 on the TWIST1 promoter region containing the putative binding site in MCF-10A cells was tested by a ChIP assay. (C) Transcriptional activity of TWIST1 promoter in the indicated cells was assessed by a dual-luciferase reporter assay. (D) TWIST1 protein expression in the indicated cells was detected by immunoblot. (E) TWIST1 protein expression in the indicated cells was detected by immunofluorescence staining. Three independent experiments were performed in triplicate. The data were expressed as means $\pm S D$. ${ }^{*} P<0.05$;

${ }^{* *} P<0.01$. ChIP, chromatin immunoprecipitation; SD, standard deviation.

inversely correlate with the FOXF2 mRNA levels in TNBC tumor (Spearman's rho $=-0.421, P=0.013$; Figure 6G). Then, the TNBC cases were divided into four groups based on FOXF2 and TWIST1 mRNA levels: FOXF2 $2_{\text {high }} / T W I S-$ $T 1_{\text {low }}(\mathrm{n}=8), \quad F O X F 2_{\text {high }} / T W I S T 1_{\text {high }}(\mathrm{n}=8), \quad F O X F 2_{\text {low }} /$ $T W I S T 1_{\text {low }}(\mathrm{n}=8)$ and $F O X F 2_{\text {low }} / T W I S T 1_{\text {high }}(\mathrm{n}=10)$. Kaplan-Meier analysis showed that the patients in the four subgroups had distinct DFS: the patients in FOXF2 $2_{\text {high }} /$ $T W I S T 1_{\text {low }}$ and $F O X F 2_{\text {low }} / T W I S T 1_{\text {high }}$ groups had the best and poorest DFS, respectively $(P=0.002$ between both); the patients in the $F O X F 2_{\text {high }} / T W I S T 1_{\text {high }}$ and $F O X F 2_{\text {low }} / T W I S T 1_{\text {low }}$ groups had moderate DFS $(P=$ 0.687 between both); $F O X F 2_{\text {high }} / T W I S T 1_{\text {low }}$ group had better DFS than $F O X F 2_{\text {low }} / T W I S T 1_{\text {low }}(P=0.063)$ and $F O X F 2_{\text {high }} / T W I S T 1_{\text {high }} \quad(P=0.143)$ groups; $F O X F 2_{\text {low }} /$ $T W I S T 1_{\text {high }}$ group had poorer DFS than $F O X F 2_{\text {high }} /$ $T W I S T 1_{\text {high }}(P=0.089)$ and $F O X F 2_{\text {low }} / T W I S T 1_{\text {low }}(P=$ 0.149 ) groups (Figure $6 \mathrm{H})$. This result suggests that
$T W I S T 1_{\text {low }}$ could contribute to the FOXF2 high-suppressed recurrence and metastasis of TNBC cells; conversely, $T W I S T 1_{\text {high }}$ could facilitate the $F O X F 2_{\text {low }}$-induced recurrence and metastasis. Taken together, these results demonstrated that TWIST1 functionally mediates the effect of FOXF2 on EMT and metastasis in BLBC cells.

\section{Discussion}

The FOX transcription factors have tissue-specific expression patterns and play critical roles in embryogenesis and tissue development through regulating tissuespecific gene expression and cell differentiation [30]. Recent evidence has demonstrated that the deregulation of FOX factors is correlated with carcinogenesis and tumor progression [21]. It is worth noting that several FOX transcription factors link to the biological characteristics of breast cancer. FOXA1 is highly expressed in luminal subtype breast cancer [31], and its deficiency promotes 

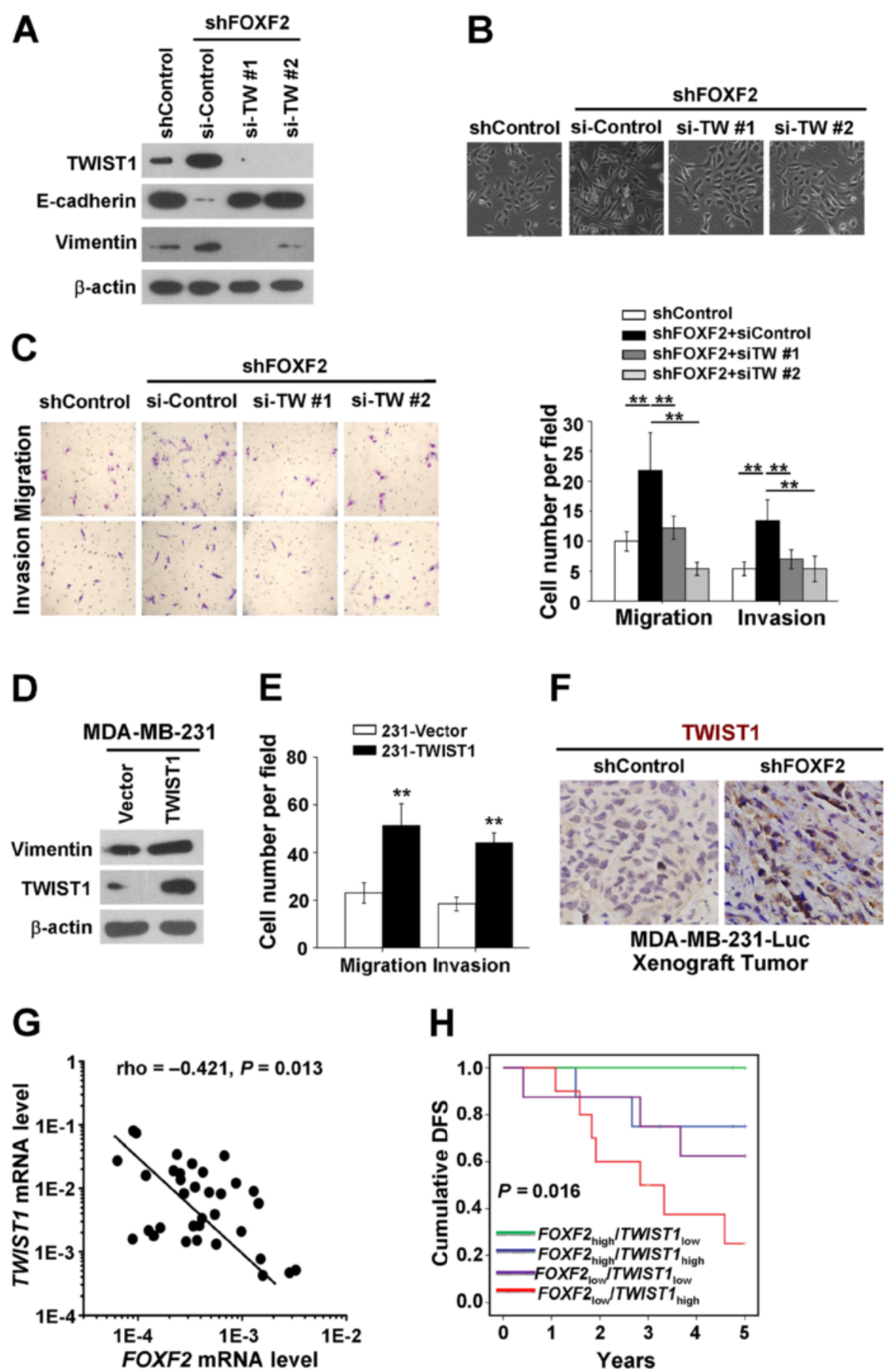

Figure 6 FOXF2 deficiency induces EMT of basal-like mammary epithelial cells via upregulation TWIST1 transcription. (A) The expression of TWIST1 and EMT markers in the indicated cells was detected by immunoblot. (B) Morphology of the indicated cells (X200). (C) Photos (Lift) and counts (Right) of migration and invasion of the indicated cells assessed by transwell assay $(\times 200)$. Three independent experiments were performed in triplicate. The data were expressed as mean \pm SD. ${ }^{* *} P<0.01$. (D) The expression of TWIST1 and vimentin in the indicated cells was detected by immunoblot. (E) The migration and invasion ability of the indicated cells were assessed by transwell assay. Three independent experiments were performed in triplicate. The data were expressed as mean \pm SD. ${ }^{* *} P<0.01$. (F) The TWIST1 protein expression in primary tumor tissues harvested from xenograft mice of MDA-MB-231-Luc shFOXF2 and MDA-MB-231-Luc shControl cells was stained by immunohistochemistry ( $\times 400)$. (G) The correlation of FOXF2 mRNA levels with TWIST1 mRNA levels in human primary TNBC tissues ( $n=34$; Spearman's correlation rho $=-0.421$, $P=0.013)$. (H) The correlation of combined FOXF2 and TWIST1 mRNA levels with the DFS of TNBC patients with FOXF2 high $/ T$ TWIST1 low $(\mathrm{n}=8)$, FOXF2 $\mathrm{high} /$ TWIST1 high $(n=8), F O X F 2_{\text {low }} / T W I S T_{\text {low }}(n=8)$ and FOXF2 low $/ T W I S T 1_{\text {high }}(n=10)$ was estimated by a Kaplan-Meier survival analysis. DFS, disease-free survival; EMT, epithelial-mesenchymal transition; SD, standard deviation; TNBC, triple-negative breast cancer. 
the metastasis of this subtype cancer [32]. FOXC1 [19], FOXC2 [4], and FOXQ1 [20] are highly expressed in basal-like subtype breast cancer, but are less present in luminal subtype cancer. The ectopic expression of these mesenchymal FOX factors in normal mammary epithelial cells is able to induce EMT and lead to an aggressive phenotype. FOXF2 is a mesenchymal transcription factor and specifically expressed in the mesenchyme adjacent to the epithelium and controls mesenchymal differentiation to maintain the tissue homeostasis via epitheliomesenchymal interactions [22]. FOXF2 was found highly expressed in benign prostatic hyperplasia and the transition zone of the normal prostate, where less suffer prostate cancer [33,34]. In this study, we showed that FOXF2 is highly expressed in most basal-like breast cells. Since basal-like breast cells are characterized by the basal gene expression and with mesenchymal characteristics [15], the expression pattern of FOXF2 in basal-like breast cells is consistent with its nature of mesenchymal transcription factors. We also found that FOXF2 deficiency promoted the metastasis of BLBC cells, which is consistent with our previous report that the under-expression of FOXF2 is associated with early-onset metastasis and poor prognosis of TNBC patients [23]. Our finding indicates that FOXF2 may play a role in controlling the differentiation state of basal-like breast cells and inhibits the aggressive property of BLBC.

It is well known that a variety of embryonic and mesenchymal transcriptional factors function as EMT activators and are involved in the aggressive behavior of BLBC, including SNAIL1 [16], SNAIL2 [17], TWIST1 [18], and FOX transcription factor family members FOXC1 [19], FOXC2 [4] and FOXQ1 [20]. Most EMTTFs were identified as activators of EMT and promoters of BLBC metastasis, while EMT suppressors are rarely identified. FOXA2 is one such rare EMT suppressor [35]. In this study, we showed that FOXF2 is a novel EMT-suppressing transcription factor in basal-like breast cells. FOXF2 deficiency enhanced the metastatic ability of basal-like mammary epithelial cells and BLBC cells by inducing an EMT phenotype. We observed that the depletion of FOXF2 results in E-cadherin and $\beta$-catenin loss from the cell membrane. It is known that during EMT progression, the loss of E-cadherin expression from adherent junction results in the release of $\beta$ catenin into the cytoplasm. In addition, we observed that the depletion of FOXF2 leads to a significant increase in $M M P s$ expression. Mesenchymal cells and the cancer cells undergoing EMT secrete extracellular matrix (ECM)-degrading enzymes to facilitate invasion and motility [36]. Matrix metalloproteinase (MMP)2 [37], MMP3 [38] and MMP9 [39] are able to induce EMT, and they trigger a positive regulatory feedback loop that stabilizes EMT. Therefore, the translocation of $\beta$-catenin translocation and upregulation of MMPs caused by FOXF2 deficiency could lead to initiate vicious cycle of EMT.

TWIST1 is recognized as a critical modulator in EMT, and its overexpression is able to trigger EMT phenotype and tumor metastasis [3]. Thus, clarifying the regulatory mechanism of TWIST1 expression would provide insight into the regulatory pathway of TWIST1-induced EMT programming and aid in developing molecular targets to inhibit tumor metastasis. It has been reported that HIF-1 [40] and p65 [41] transcriptionally activate TWIST1 expression to mediate hypoxia-induced and tumor necrosis factor alpha (TNF- $\alpha$ )-induced EMT and tumor metastasis, respectively. miR-720 [42] and miR106b [43] suppress TWIST1 expression by posttranscriptional regulatory mechanisms. In this study, we identified FOXF2 as a transcriptional suppressor of TWIST1. Therefore, we speculate that the two embryonic/mesenchymal transcription factors might act cooperatively to maintain tissue homeostasis through balancing the differentiation or dedifferentiation of mesenchymal/myoepithelial cells. FOXF2 deficiency could lead to more dedifferentiation of myoepithelial cells and an aggressive phenotype in BLBC via a TWIST1mediated pathway. This novel finding provided an unanticipated regulatory pathway of TWIST-induced EMT and metastasis in BLBC.

In EMT programming, pleiotropic EMT-TFs form an interaction network to enable them to work in concert to regulate the EMT phenotype. The zinc-finger transcription factors SNAIL1 [44], SNAIL2 [45], ZEB1 [46] and ZEB2 [47] directly bind to the E-boxes of the $C D H 1$ promoter to repress its transcription. TWIST1 [3], Goosecoid [48], and FOXC2 [4] trigger EMT by indirectly suppressing $C D H 1$ transcription. TWIST1 directly regulates SNAIL2 transcription, and SNAIL2 is an essential mediator of TWIST1-induced EMT [49]. TWIST1 can also induce FOXC2 and SNAIL2 expression [7]. SNAIL1 has been found to activate ZEB1 [2], FOXC2 and SNAIL2 expression [7]. In this study, we found that FOXF2 not only negatively regulated TWIST1 expression but also regulated the expression of SNAIL1 and SNAIL2, ZEB1, $Z E B 2$ and GSC in basal-like cell lines. Interestingly, FOXF2 regulated TWIST1 expression in all the three cell lines, but only regulated SNAIL2 in MCF-10A and BT549 cells, regulated ZEB1 and GSC in MDA-MB-231 and BT-549 cells, regulated ZEB2 in MCF-10A and MDA-MB-231 cells. Therefore, in addition to activating TWIST1 transcription, FOXF2 deficiency may induce EMT through orchestrating different EMT-TFs expression in the different basal-like breast cells.

In addition to BLBC, we also tested the effect of FOXF2 on luminal breast cancer cells. However, we observed that the ectopic expression of FOXF2 could not significantly change the phenotype of luminal breast 
cancer cells, even slightly enhance the migration and invasion, and slightly increase the transcription of TWIST1. Our results suggest that there are different functions of FOXF2 in basal-like and luminal cells. Based on our current results, we speculate that mesenchymal FOXF2 might be induced expression in luminal cells that had undergone transdifferentiation into basal-like cells, and when FOXF2 depletion during the progression of the cells adapted mesenchymal characteristics could drive these cells to possess more mesenchymal stem-like plasticity and aggressive phenotype by activating the transcription of target gene TWIST1 and upregulating the expression of other EMT-TFs. The regulatory mechanisms of the different expression pattern and functions of FOXF2 in basal-like and luminal breast cancer cells should be further deeply investigated. Since FOXF2 plays an essential role in the metastasis of BLBC cells, the exogenous administration of FOXF2 or interference of FOXF2-TWIST1 pathway may be a promising therapeutic strategy for improving the outcome of BLBC patients.

\section{Conclusions}

In conclusion, our study identified FOXF2 as a novel EMT-suppressing transcription factor in BLBC. FOXF2 deficiency enhances metastatic ability of BLBC cells via activating an EMT program by upregulating the transcription of TWIST1.

\section{Additional file}

Additional file 1: Figure S1. FOXF2 overexpression was incapable of inducing EMT of luminal breast cancer cells. (A) The protein expression of FOXF2 in the indicated cells was detected by immunoblot. (B) The migration and invasion ability of the indicated cells were assessed by transwell assay. (C) The proliferation ability of the indicated cells was determined by MTT assay. (D) Morphological photos of the indicated cells ( $\times 200)$. (E) The protein expression of EMT markers in the indicated cells was detected by immunoblot. (F) The mRNA expression levels of EMT-TFS SNAIL1, SNALI2, TWIST1 and GSC in the indicated cells were measured by RT-qPCR. Fold changes were relative to the mRNA expression of the control cells. (G) Transcriptional activity of TWIST1 promoter in the indicated cells was assessed by a dual-luciferase reporter assay. Three independent assays were performed in triplicate. The data were expressed as mean \pm SD. n.S., $P>0.05$.

\section{Abbreviations}

bHLH: basic helix-loop-helix; BLBC: basal-like breast cancer; ChIP: chromatin immunoprecipitation; CK: cytokeratins; DFS: disease-free survival; ECM: extracellular matrix; EGFR: epithelial growth factor receptor; EMT: epithelial-mesenchymal transition; EMT-TFs: EMT-transcription factors; ER: estrogen receptor; FBS: fetal bovine serum; FOX: forkhead box; GAPDH: glyceraldehyde 3-phosphate dehydrogenase; GOBO: Gene expression-based Outcome for Breast cancer Online; H\&E: hematoxylin and eosin; HER2: human epidermal growth factor receptor 2; MMP: matrix metalloproteinase; MTT: 3-(4,5-dimethylthiazol-2-yl)-2,5-diphenyltetrazolium bromide; PBS: phosphate-buffered saline; PR: progesterone receptor; RT-qPCR: reverse transcription quantitative PCR; SCID: severe combined immunodeficiency; SD: standard deviation; shRNA: short hairpin RNA; siRNA: small interfering RNA; TMUCIH: Tianjin Medical University Cancer Institute and Hospital; TNBC: triple-negative breast cancer; TNF-a: tumor necrosis factor alpha; TSS: transcription start site.

\section{Competing interests}

The authors declare that they have no competing interests.

\section{Authors' contributions}

QSW participated in the study design, performed experiments, analyzed and interpreted data, and wrote the manuscript. PZK participated in the study design and performed experiments. XQL and FY contributed to acquisition of data and revising the manuscript critically for important intellectual content. YMF conceived the study, interpreted data, and wrote the manuscript. All authors read and approved the final manuscript.

\section{Acknowledgements}

This work was supported by the National Natural Science Foundation of China (No. 30872518, No. 81272357, No. 81201652 and No. 81472680) and the Major Program of Applied Basic Research Projects of Tianjin (No. 09JCZDJC19800 and No. 13JCZDJC30100).

Received: 14 August 2014 Accepted: 4 February 2015

Published online: 26 February 2015

\section{References}

1. Jemal A, Bray F, Center MM, Ferlay J, Ward E, Forman D. Global cancer statistics. CA Cancer J Clin. 2011;61:69-90.

2. Thiery JP, Acloque H, Huang RY, Nieto MA. Epithelial-mesenchymal transitions in development and disease. Cell. 2009;139:871-90.

3. Yang J, Mani SA, Donaher JL, Ramaswamy S, Itzykson RA, Come C, et al. Twist, a master regulator of morphogenesis, plays an essential role in tumor metastasis. Cell. 2004;117:927-39.

4. Mani SA, Yang J, Brooks M, Schwaninger G, Zhou A, Miura N, et al. Mesenchyme Forkhead 1 (FOXC2) plays a key role in metastasis and is associated with aggressive basal-like breast cancers. Proc Natl Acad Sci U S A. 2007;104:10069-74

5. Thiery JP. Epithelial-mesenchymal transitions in tumour progression. Nat Rev Cancer. 2002;2:442-54.

6. Graham TR, Zhau HE, Odero-Marah VA, Osunkoya AO, Kimbro KS, Tighiouart $\mathrm{M}$, et al. Insulin-like growth factor-I-dependent up-regulation of ZEB1 drives epithelial-to-mesenchymal transition in human prostate cancer cells. Cancer Res. 2008;68:2479-88.

7. Taube JH, Herschkowitz Jl, Komurov K, Zhou AY, Gupta S, Yang J, et al. Core epithelial-to-mesenchymal transition interactome gene-expression signature is associated with claudin-low and metaplastic breast cancer subtypes. Proc Natl Acad Sci U S A. 2010;107:15449-54.

8. Firulli $A B$, Conway SJ. Phosphoregulation of Twist1 provides a mechanism of cell fate control. Curr Med Chem. 2008;15:2641-7.

9. Leptin M. Twist and snail as positive and negative regulators during Drosophila mesoderm development. Genes Dev. 1991;5:1568-76.

10. Thisse B, el Messal M, Perrin-Schmitt F. The twist gene: isolation of a Drosophila zygotic gene necessary for the establishment of dorsoventral pattern. Nucleic Acids Res. 1987;15:3439-53.

11. Fu J, Qin L, He T, Qin J, Hong J, Wong J, et al. The TWIST/Mi2/NuRD protein complex and its essential role in cancer metastasis. Cell Res. 2011;21:275-89.

12. Toft DJ, Cryns VL. Minireview: basal-like breast cancer: from molecular profiles to targeted therapies. Mol Endocrinol. 2011;25:199-211.

13. Carey L, Winer E, Viale G, Cameron D, Gianni L. Triple-negative breast cancer: disease entity or title of convenience? Nat Rev Clin Oncol. 2010;7:683-92.

14. Metzger-Filho O, Tutt A, de Azambuja E, Saini KS, Viale G, Loi S, et al. Dissecting the heterogeneity of triple-negative breast cancer. J Clin Oncol. 2012;30:1879-87

15. Neve RM, Chin K, Fridlyand J, Yeh J, Baehner FL, Fevr T, et al. A collection of breast cancer cell lines for the study of functionally distinct cancer subtypes. Cancer Cell. 2006;10:515-27.

16. Dong C, Yuan T, Wu Y, Wang Y, Fan TW, Miriyala S, et al. Loss of FBP1 by Snail-mediated repression provides metabolic advantages in basal-like breast cancer. Cancer Cell. 2013;23:316-31.

17. Storci G, Sansone P, Trere D, Tavolari S, Taffurelli M, Ceccarelli C, et al. The basal-like breast carcinoma phenotype is regulated by SLUG gene expression. J Pathol. 2008;214:25-37.

18. Li S, Kendall SE, Raices R, Finlay J, Covarrubias M, Liu Z, et al. TWIST1 associates with NF-kappaB subunit RELA via carboxyl-terminal WR domain to promote cell autonomous invasion through IL8 production. BMC Biol. $2012 ; 10: 73$. 
19. Ray PS, Wang J, Qu Y, Sim MS, Shamonki J, Bagaria SP, et al. FOXC1 is a potential prognostic biomarker with functional significance in basal-like breast cancer. Cancer Res. 2010;70:3870-6.

20. Qiao Y, Jiang X, Lee ST, Karuturi RK, Hooi SC, Yu Q. FOXQ1 regulates epithelial-mesenchymal transition in human cancers. Cancer Res. 2011;71:3076-86.

21. Myatt SS, Lam EW. The emerging roles of forkhead box (Fox) proteins in cancer. Nat Rev Cancer. 2007;7:847-59.

22. Aitola M, Carlsson P, Mahlapuu M, Enerback S, Pelto-Huikko M. Forkhead transcription factor FoxF2 is expressed in mesodermal tissues involved in epithelio-mesenchymal interactions. Dev Dyn. 2000;218:136-49.

23. Kong PZ, Yang F, Li L, Li XQ, Feng YM. Decreased FOXF2 mRNA expression indicates early-onset metastasis and poor prognosis for breast cancer patients with histological grade II tumor. PLoS One. 2013;8:e61591.

24. Hirata H, Ueno K, Shahryari V, Deng G, Tanaka Y, Tabatabai ZL, et al. MicroRNA-182-5p promotes cell invasion and proliferation by down regulating FOXF2, RECK and MTSS1 genes in human prostate cancer. PLoS One. 2013:8:e55502.

25. Shi W, Gerster K, Alajez NM, Tsang J, Waldron L, Pintilie M, et al. MicroRNA301 mediates proliferation and invasion in human breast cancer. Cancer Res. 2011;71:2926-37.

26. Yu Y, Xiao CH, Tan LD, Wang QS, Li XQ, Feng YM. Cancer-associated fibroblasts induce epithelial-mesenchymal transition of breast cancer cells through paracrine TGF-beta signalling. Br J Cancer. 2014;110:724-32.

27. Wang CA, Jedlicka P, Patrick AN, Micalizzi DS, Lemmer KC, Deitsch E, et al. SIX1 induces lymphangiogenesis and metastasis via upregulation of VEGF-C in mouse models of breast cancer. J Clin Invest. 2012;122:1895-906.

28. Faustino-Rocha A, Oliveira PA, Pinho-Oliveira J, Teixeira-Guedes C, SoaresMaia R, da Costa RG, et al. Estimation of rat mammary tumor volume using caliper and ultrasonography measurements. Lab Anim. 2013;42:217-24.

29. Ringner M, Fredlund E, Hakkinen J, Borg A, Staaf J. GOBO: gene expressionbased outcome for breast cancer online. PLoS One. 2011;6:e17911.

30. Hannenhalli S, Kaestner KH. The evolution of Fox genes and their role in development and disease. Nat Rev Genet. 2009;10:233-40.

31. Perou CM, Sorlie T, Eisen MB, van de Rijn M, Jeffrey SS, Rees CA, et al. Molecular portraits of human breast tumours. Nature. 2000:406:747-52.

32. Bernardo GM, Bebek G, Ginther CL, Sizemore ST, Lozada KL, Miedler JD, et al. FOXA1 represses the molecular phenotype of basal breast cancer cells. Oncogene. 2013;32:554-63.

33. van der Heul-Nieuwenhuijsen $L$, Hendriksen PJ, van der Kwast TH, Jenster G. Gene expression profiling of the human prostate zones. BJU Int. 2006;98:886-97.

34. van der Heul-Nieuwenhuijsen L, Dits NF, Jenster G. Gene expression of forkhead transcription factors in the normal and diseased human prostate. BJU Int. 2009;103:1574-80.

35. Tang $Y$, Shu G, Yuan $X$, Jing N, Song J. FOXA2 functions as a suppressor of tumor metastasis by inhibition of epithelial-to-mesenchymal transition in human lung cancers. Cell Res. 2011;21:316-26.

36. Taddei ML, Giannoni E, Comito G, Chiarugi P. Microenvironment and tumor cell plasticity: an easy way out. Cancer Lett. 2013;341:80-96.

37. Duong TD, Erickson CA. MMP-2 plays an essential role in producing epithelial-mesenchymal transformations in the avian embryo. Dev Dyn. 2004;229:42-53.

38. Radisky DC, Levy DD, Littlepage LE, Liu H, Nelson CM, Fata JE, et al. Rac1b and reactive oxygen species mediate MMP-3-induced EMT and genomic instability. Nature. 2005;436:123-7.

39. Tan TK, Zheng G, Hsu TT, Wang Y, Lee WW, Tian X, et al. Macrophage matrix metalloproteinase-9 mediates epithelial-mesenchymal transition in vitro in murine renal tubular cells. Am J Pathol. 2010;176:1256-70.

40. Yang MH, Wu MZ, Chiou SH, Chen PM, Chang SY, Liu CJ, et al. Direct regulation of TWIST by HIF-1alpha promotes metastasis. Nat Cell Biol. 2008;10:295-305

41. Li CW, Xia W, Huo L, Lim SO, Wu Y, Hsu JL, et al. Epithelial-mesenchymal transition induced by TNF-alpha requires NF-kappaB-mediated transcriptional upregulation of Twist1. Cancer Res. 2012;72:1290-300.

42. Li LZ, Zhang CZ, Liu LL, Yi C, Lu SX, Zhou X, et al. miR-720 inhibits tumor invasion and migration in breast cancer by targeting TWIST1. Carcinogenesis. 2014; 35:469-78.

43. Dong P, Kaneuchi M, Watari H, Sudo S, Sakuragi N. MicroRNA-106b modulates epithelial-mesenchymal transition by targeting TWIST1 in invasive endometrial cancer cell lines. Mol Carcinog. 2014;53:349-59.
44. Batlle E, Sancho E, Franci C, Dominguez D, Monfar M, Baulida J, et al. The transcription factor snail is a repressor of E-cadherin gene expression in epithelial tumour cells. Nat Cell Biol. 2000;2:84-9.

45. Hajra KM, Chen DY, Fearon ER. The SLUG zinc-finger protein represses E-cadherin in breast cancer. Cancer Res. 2002;62:1613-8.

46. Eger A, Aigner K, Sonderegger S, Dampier B, Oehler S, Schreiber M, et al. DeltaEF1 is a transcriptional repressor of E-cadherin and regulates epithelial plasticity in breast cancer cells. Oncogene. 2005;24:2375-85.

47. Comijn J, Berx G, Vermassen P, Verschueren K, van Grunsven L, Bruyneel E, et al. The two-handed $\mathrm{E}$ box binding zinc finger protein SIP1 downregulates E-cadherin and induces invasion. Mol Cell. 2001;7:1267-78.

48. Hartwell KA, Muir B, Reinhardt F, Carpenter AE, Sgroi DC, Weinberg RA. The Spemann organizer gene, Goosecoid, promotes tumor metastasis. Proc Natl Acad Sci U S A. 2006;103:18969-74.

49. Casas E, Kim J, Bendesky A, Ohno-Machado L, Wolfe CJ, Yang J. Snail2 is an essential mediator of Twist1-induced epithelial mesenchymal transition and metastasis. Cancer Res. 2011;71:245-54.

\section{Submit your next manuscript to BioMed Central and take full advantage of:}

- Convenient online submission

- Thorough peer review

- No space constraints or color figure charges

- Immediate publication on acceptance

- Inclusion in PubMed, CAS, Scopus and Google Scholar

- Research which is freely available for redistribution

Submit your manuscript at www.biomedcentral.com/submit 